\title{
Ist Fingerknacken schädlich?
}

\section{Die Angewohnheit, die Fingergelenke knacken zu lassen, hat wohl keine funktionelle Relevanz, zumindest nicht kurzfristig. Zu diesem Ergebnis kommt eine Studie aus Kalifornien, in der 400 Gelenke unmittelbar vor und nach dem Knacken untersucht wurden.}

_ 40 asymptomatische Freiwillige, von denen 30 in der Lage waren, ihre Metakarpophalangealgelenke knacken zu lassen, nahmen an der Studie teil. Alle Probanden mussten ihre Finger am Grundgelenk auseinanderziehen. Sowohl vor als auch nach dem Versuch wurden sie auf Schwellungen und Griffstärke der Hand sowie auf Beweglichkeit der Fingergelenke untersucht. Per Ultraschall wurde zudem in Echtzeit beobachtet, was während des Fingerknackens geschah.

Insgesamt zeigten sich keine funktionellen Unterschiede zwischen Knackern und Nicht-Knackern. Auch bei der mittels Dynamometer gemessenen Griffstärke ergab sich kein nennenswerter Unterschied zwischen den Gruppen. Schwellungen wurden in beiden Grup-

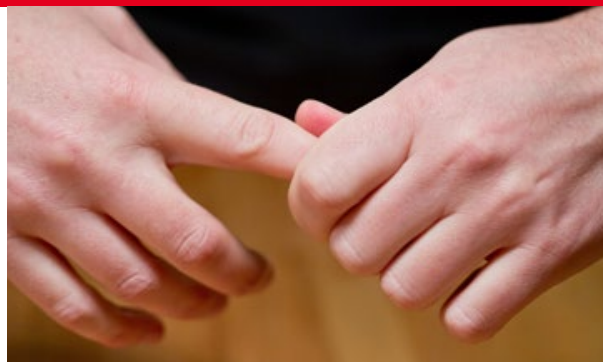

Unter dem Knacken leiden v. a. die Anderen.

pen nicht beobachtet. Im Ultraschall war das Knacken stets begleitet von einem sichtbaren Phänomen. Die Untersucher beschreiben dies als ein „wie aus dem Nichts auftretendes hyperechogenes Aufleuchten im Gelenk“.

Was das Fingerknacken langfristig mit den Gelenken macht, insbesondere, ob etwa ein erhöhtes Arthroserisiko besteht, bleibt zu untersuchen. = eo

- Boutin RD et al. Clin Orthop Relat Res 2017;475:1265-1271

\section{Leptin als „Missing Link“?}

\section{Übergewichtige Mädchen pubertieren früher}

\begin{abstract}
Übergewicht im Kindesalter führt bei Mädchen zu einem früheren Einsetzen der Pubertät. Zu dicke Jungen pubertieren dagegen eher später. Wissenschaftler der Uni Witten-Herdecke haben mögliche Gründe dafür gefunden.
\end{abstract}

_ Teilnehmer ihrer Studie waren 160 übergewichtige Kinder, bei denen die Pubertät noch nicht eingesetzt hatte. Die Mädchen waren bei Studienbeginn zwischen neun und elf Jahre alt, die Jungen zwischen elf und dreizehn. Kinder mit einem BMI über der 90. altersentsprechenden Perzentile wurden als übergewichtig definiert. Als adipös galten Kinder, wenn ihr BMI jenseits der 97. Perzentile lag.

Alle Kinder nahmen ein Jahr lang am sog. „Obeldicks“-Pro- gramm teil, einer ambulanten Intervention mit dem Ziel einer nachhaltigen Lebensstiländerung. Vor und nach der Intervention bestimmten die Forscher neben Körpergröße und BMI verschiedene Stoffwechselparameter sowie bei Jungen das Genitalstadium und bei Mädchen das Stadium des Brustwachstums.

Von Interesse war vor allem, ob die Intervention Einfluss auf den Pubertätsbeginn hatte. Mädchen, die erfolgreich abgenommen hatten, kamen signifikant später in die $\mathrm{Pu}$ bertät als Mädchen mit stabilem BMI. Während Letztere im Studienzeitraum mit einer Wahrscheinlichkeit von $75 \% \mathrm{zu}$ pubertieren begannen, war das bei den erfolgreichen „Obeldicks“-Teilnehmerinnnen nur zu rund $46 \%$ der Fall.
Bei den Jungen führte die Gewichtsabnahme dagegen $\mathrm{zu}$ einem früheren Pubertätsbeginn: Rund 77\% der Jungen, die es geschafft hatten abzunehmen, wurden innerhalb eines Jahres geschlechtsreif; bei den Jungen ohne BMIReduktion waren es nur knapp 54\%.

Wie die Autoren berichten, war der Pubertätsbeginn mit einer Stimulation der Hypothalamus-Hypophysen-Gonaden-Achse verknüpft. Sowohl die LHund FSH-Werte als auch der LH/FSHQuotient stiegen bei beiden Geschlechtern an. Bedeutung hat dabei offenbar das im Fettgewebe produzierte Leptin. Die Leptinspiegel sinken, wenn adipöse Kinder abnehmen. Andererseits konnte gezeigt werden, dass sie speziell bei Mädchen vor Pubertätsbeginn ansteigen und dass dies mit dem Anstieg der Gonadotropine LH und FSH korreliert. - eo

- Reinehr Tet al. JPEDS, online 24. Februar 2017 doi: 10.1016/j.jpeds.2017.01.066 